\title{
Design and Manufacturing Process of Micro Air Vehicles
}

\author{
Mateusz Rakiec , Aleksandra Kwiecien , Rafal Muchowski and Bartosz Kazmierski \\ Rzeszow University of Technology, Powstancow Warszawy Av. 12, 35-959 Rzeszow, Poland
}

\begin{abstract}
This paper shows shortly the designing, optimization and manufacturing process of light and compact, carbon fiber MAV. It also covers testing and results. The main purpose was to make an innovative prototype of UAV which is easy to transport, assembly and carry as much high volume payload as possible. The optimization process started from defining the constrains. To gain information needed to choose the best solution and perform optimization, authors made many tests. The material, which was chosen, had to be as light and durable as possible - this characteristics were later checked in tensile test of carbon fiber samples. The main optimization algorithm do initial research about the rest parameters of aircraft. The point was to narrow the range of analyzed chord's values and find the best motors. For better performance the original airfoil was developed. It works perfectly in low Reynolds number area and has perfect characteristics for high-lift airfoils, compared to similar. It was later checked in CFD. The special manufacturing process of carbon fiber airplane was designed. After all the construction was tested. The successful flight tests confirmed that design process was appropriate and done well. All theoretical assumptions were also right.
\end{abstract}

\section{Initial Considerations}

The main objective of this paper is to design a small, light-weight unmanned aerial vehicle, capable of lifting the greatest possible payload compared to empty mass. It should carry high volume payload and be assembled quickly from the small package. The designing process of heavy UAVs is well developed these days. There are also plenty of publications about design mini unmanned air vehicles but they mainly cover the quadcopter and similar systems and those about fixed-wing constructions, which are very light, could be back-packed, assembled and deployed by just one person, are not well developed [1-2]. The most important aspects to find best solution which meet the criteria set above are:

- make an MAV which will be as light as possible but also durable,

- aircraft must be hand launched by one person and land on the ground

- construct the airplane, which will be packed in the box with small dimension and be easy to assemble

- design the aircraft as safe and simple as possible.

To make it possible, was planned:

- make a carbon fiber, single engine UAV with the lowest empty mass, which fits in the box,

- develop software to optimize the construction for best performance,

- find appropriate materials - light but durable and design best manufacturing process.

The main problem to discuss was the dimension constraints imposed by box dimensions. The optimization software was developed to deal with the problem and to analyze various range of size configurations very thoroughly.

\section{Final Conception}

The main limitations of constructions are dimensions of packing box, assembly time and payload's requirements. Also that the maximum dimension of single piece caused by box is $35 \mathrm{~cm}$, maximum payload size is $10 \mathrm{~cm} \mathrm{x} 10 \mathrm{~cm} \mathrm{x}$ $35 \mathrm{~cm}$, wing chord between $9,9 \mathrm{~cm}$ and $25,8 \mathrm{~cm}$ and maximum angle of attack $8^{\circ}$.

The number of wing sections that could be possibly fitted inside the box is three. This impose the constraint of wingspan about $1 \mathrm{~m}$ depending on dihedral. The wingspan limitation suggested using rectangular, no swept wing to obtain the largest surface, that seemed to be the key in high lifting capacity.

For best configuration selection the discriminators were defined with specified importance and differentiated from each other. Many ideas were considered and in the end conventional configuration was chose. It is light and easily controllable as long as designed properly, provides fast loading and assembly possibilities. Aircraft is also predictable and, due to extra stability, the payload might be loaded over the wing.

To find the dihedral configuration the CFD analysis was performed to assess whether the expected growth in induced drag, due to the shape, has enough small impact to remain rectangular wing assumption. The collected data by ANSYS software confirmed the benefit of above ascertainment. Two options of wing dihedral configuration were took into consideration. The analysis 
suggested application of double dihedral configuration for best lift to drag ratio and simple assembly.

When discussing the issue of payload layout and its fixing, many variants were took into consideration.

The choice was between the payload mounted over the wing and under it, with lowest wing area covered. Basing on the fact that landing vulnerability is more essential than maneuverability and avoiding the payload damage the payload mounted over the wing was chosen.

\section{Airfoil Selection}

The worst part with finding best airfoil was to deal with low Reynolds numbers area. Every known high lift coefficient characteristic wasn't appropriate for particular values of Reynolds number around 100000 , so the new one was designed [3-6]. After analysis in XFLR5 the RML (red line on Figure 1) was developed, which fits perfectly for the main purposes. It has linear $C_{L}(\alpha)$ ratio and, compared to other airfoils, higher critical angle of attack. This makes construction safer - smooth stall characteristics is placed at about $12^{\circ}$. Also airfoil's $C_{L}\left(C_{D}\right)$ ratio is much better and makes an aircraft more stable. Moreover the drag coefficient is lower. The figures show that in selected Re numbers area many airfoils' $C_{L}$ vs $C_{D}$ dependence is irregular, which is an adverse. It means that pilot could have problem with control an aircraft during take-off and landing. Whole research has proved that design process was right. It was later checked in ANSYS software.
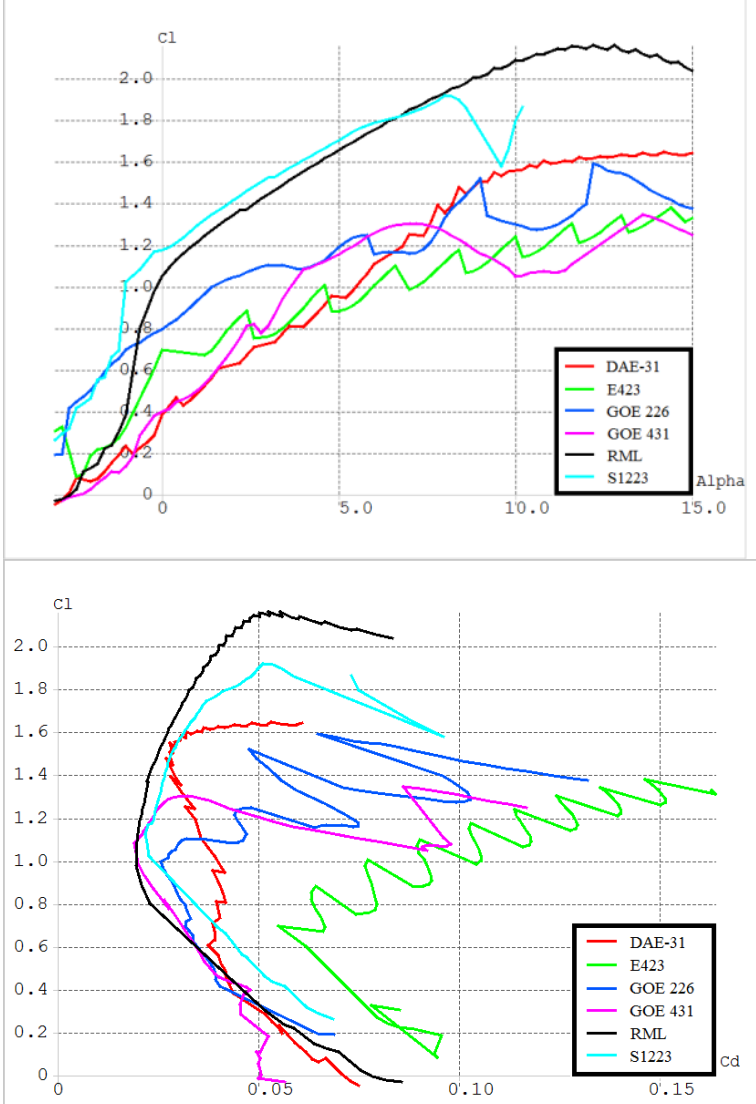

Figure 1. Airfoil comparison

\section{Optimization}

\subsection{Initial Performance Considerations}

The generic algorithm in Matlab software was developed to do initial research about what size of electric motor will probably be used and what the estimated range of chord dimension was. The additional constraint, resulting from the algorithm structure, was the limit for maximum motor. The input of the application was the airfoil deducted by aerodynamic research. The gradually increased variables were: wing's chord, an angle of attack and dynamic thrust approximation. The point was to narrow the range of analyzed chord's values and to decide what motors should be used in some detailed tests.

\subsection{Optimization Process}

Since the crucial data was collected, an exact size of wing and its angle of attack was calculated. The block diagram of second, final optimization software is in Figure 2.

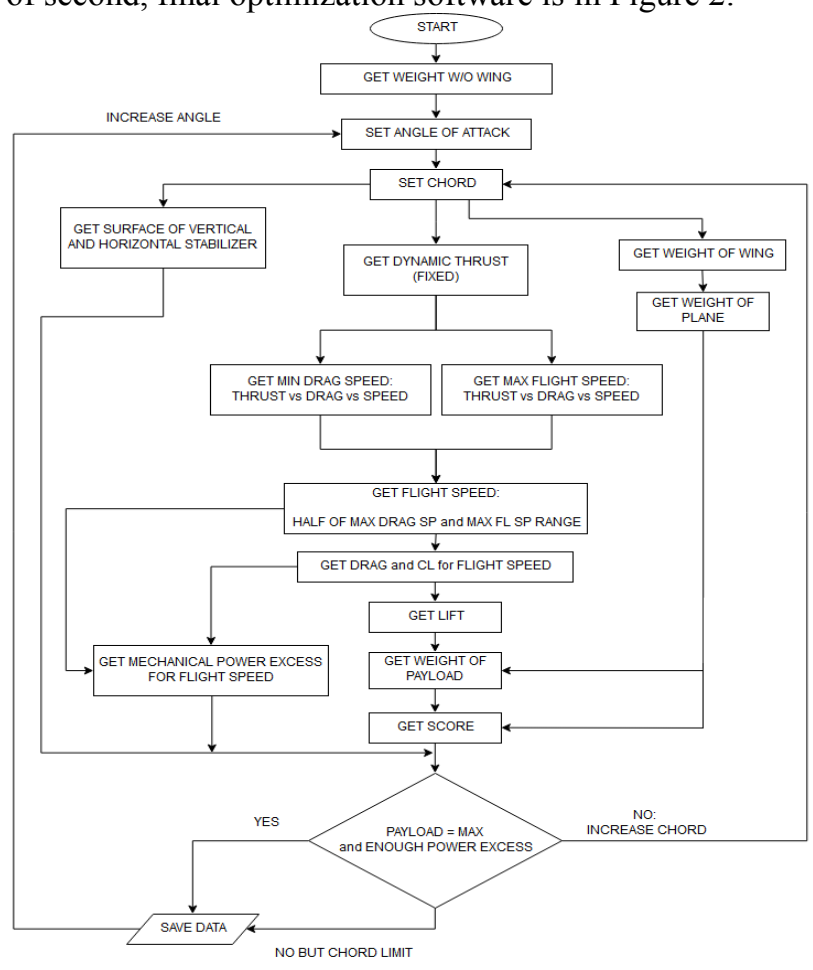

Figure 2. Final optimization loop

The variable list was divided into major variable - chord of wing, and the minor variable - angle of attack. Both of them were increased step by step to make sure to not omit any solution [7]. The algorithm helped to get information about the size of horizontal and vertical stabilizers. It was also necessary to arrange the way of flight speed deduction. The application calculated the corresponding values of $\mathrm{C}_{1}, \mathrm{C}_{\mathrm{d}}$, Reynolds number and total drag depending on velocity, including parasite drag, induced drag, drag generated by fuselage, empennage, fittings and so on. The issue, which had to be solved, was what value of airplane's speed to be used for further calculations. The maximum flight speed and minimum drag speed were found by transforming drag and thrust data into mechanical power data [2]. The decision was to use the 
exact middle value from range between the minimum drag speed and maximum flight speed. Also next criteria was defined to have at least $20 \%$ of mechanical power excess, defined as the ratio of difference between the power available and power required to power required. The reason was to ensure possibility to climb or remain constant altitude of flight in poor weather conditions and to compensate possible manufacturing inaccuracies.

Determined value of flight speed, lift coefficient and wing surface were used to calculate the lift [8]. The difference between the lift and empty weight of the aircraft multiplied by safety factor, which was assumed to be equal to 0.9 , expressed the weight of payload possible to carry by given parameters configuration. The application of safety factor results from the risk of momentary unpredicted flight issues and risk of calculation method deviation. The algorithm was checking whether the airplane could carry the predicted payload of weight 1.4 kilograms and whether there is at least $20 \%$ of mechanical power excess. Meeting these both conditions or lack of further values of chord from range $9.9-25.8 \mathrm{~cm}$ made the data was saved on hard disk for analysis and angle of attack was increased. In such case the process was performed again. Otherwise, the chord was increased without rising an angle. The maximum optimization performance was reached for maximum angle of attack $\left(8^{\circ}\right)$ and chord $11 \mathrm{~cm}$ but there was also growth for $5^{\circ}$. After taking into consideration that optimal angle of attack, according to the aerodynamic research, is $2.5^{\circ}$ and basing on the above plots increasing this angle ensures higher score due to lower weight of empty MAV. Application of such great angle as $8^{\circ}$ will make the flight hazardous because of potential risk of stall or shortage of controllability. The compromise between those two aspects, it was decided to choose $3.5^{\circ}$ angle of attack and $16 \mathrm{~cm}$ of chord. Longer chord will cause problems with placement of the airplane inside of the box. Low extension of the sought values should not bring a significant lose in competition results but will make predictability of flight higher. This extension was another, the third optimization precaution, since awareness of the risk of calculations deviation came from slight simplifications. An angle of attack of the wing was adjusted by the pilot during flight and lift force generated by horizontal stabilizer is changed by the deflection of elevator, that is why the suggested value of angle does not have to be strictly followed. However, deviation from calculated value cannot be too excessive because of additional growth in drag generated by fuselage and tail. That is why angle of attack is a secondary variable and is meant to be a clue for further design and analysis how much the change in the angle influences the maximum payload. Another purpose of this was to show particularly beneficial range of angles of attack if such exists.

\section{Structural Design}

By using composites it was possible to make light and durable spar. Spar flange is made of carbon fibre filaments and Rohacell inside. Because carbon roving has enormous strength, classic calculations were useless. Minimum cross section of spar was defined by the means of bending moment (taken from XFLR5) that was converted to tensile and compressive forces in the box spar in every segment [9]. The stiffness of a material is the most important in this technology so the tensile testing on Zwick/Roell Z100 machine were performed. Many different carbon fibre samples were made and tested to find those which meets load requirements for spar flange.

\section{Analysis}

\subsection{Dynamic \& Static Stability}

The static stability was tested with full payload onboard in AVL, at airspeed predicted for landing phase equals to $v=v_{\text {stall }} * 1.2=8.6 \frac{\mathrm{m}}{\mathrm{s}}$. It showed that static margin of $17 \%$ was enough for good longitudinal static stability. Dynamic stability was analyzed in a similar manner, with fully loaded aircraft's moments of inertia obtained via CAD model. Every point of the system was in upper left quadrant of a plot, with negative real components, which means that each of the five modes are stable.

\subsection{Horizontal \& Vertical Stabilizer}

The next point was to define the type of fuselage. Since there is limited space it was decided to choose straightpipe frame divided into 4 parts. Also was important to make a choice of tail type and empennage. Many configurations were analyzed and two of them were found to be the best ones: fuselage mounted stabilizers and X-tail empennage. The decision between those two options was based on the fact that torque that will occur takes low value, while landing vulnerability is more meaningful. The fuselage mounted stabilizers was the final choice [8].

\subsection{Computional Fluid Dynamics verification}

After design original airfoil, it was decided to verify it and do more accurate CFD analysis as shown in Figure 3. Whole aircraft geometry was created in ANSYS software to get as accurate results as possible and to check if the airfoil achieves characteristics like in the vortex lattice method. To find laminar to turbulent transition in boundary layer the Transition SST turbulence model was used. This analysis affirmed previous calculations, so the optimization was correct. 


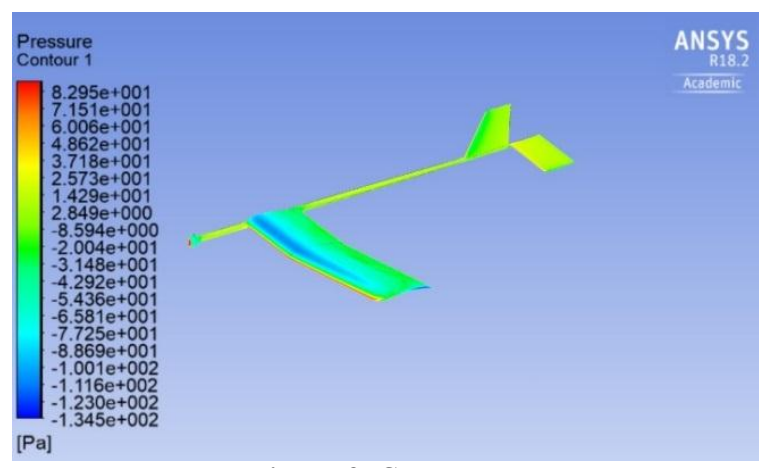

Figure 3. CFD output

\subsection{Power Plant Research}

One of the most important test was to find the best power plant. The results showed that about $140 \mathrm{~W}$ electric motor will probably get the best results. The three various motors were selected to perform thorough dynamic thrust tests in wind tunnel, providing for margin of error of algorithm approximations:

- Emax RS2306 2400 RPM/V 200W

- DRC Inferno Motor 2207.5 - 2550 RPM/V 200W

- Axi 2208/26 V2 - $1420 \mathrm{RPM} / \mathrm{V}$ - 130W

The data collected was concerned, apart from thrust decrement by growth of airflow speed, electric and temperature performance [2]. The final choice was the Axi 2208/26 due to:

- the lowest value of slope of dynamic thrust curve,

- the lowest power consumption that allows to reduce weight by using less capacity battery,

- the lowest temperature in similar conditions reducing risk of in-flight breakdown

Since the crucial data was collected, it was possible to calculate an exact size of wing and its angle of attack. The second, final optimization software was prepared.

\section{Manufacturing and Flight Tests}

To make the construction as light as possible it was decided to make it entirely of carbon fiber. Molds were designed and milled for whole aircraft. Wings consist of three, easy to assemble parts and ae made in sandwich technology. Fuselage is seamless, made of halves, glued together under pressure. Stabilizers were made in full core technology using light foam, covered with biaxial carbon fiber with basis weight of 20 grams per square meter. Every element was post cured in temperature $80^{\circ} \mathrm{C}$ for minimum 15 hours, after initial cure. This process considerably improves strength and heat resistance.

The aircraft ready to fly, without payload, weighs only 278 grams.

As a result of the research the flight tests were performed as shown in Figure 4. They confirmed that developed optimization and manufacturing process was appropriate and done well. All theoretical assumptions were also correct. The airplane stably flied with full assumed payload with dimensions of $10 \mathrm{~cm} \times 10 \mathrm{~cm} \times 35 \mathrm{~cm}$, weighing 1.4 kilograms. The flight tests were done in different weather conditions and payload amount.
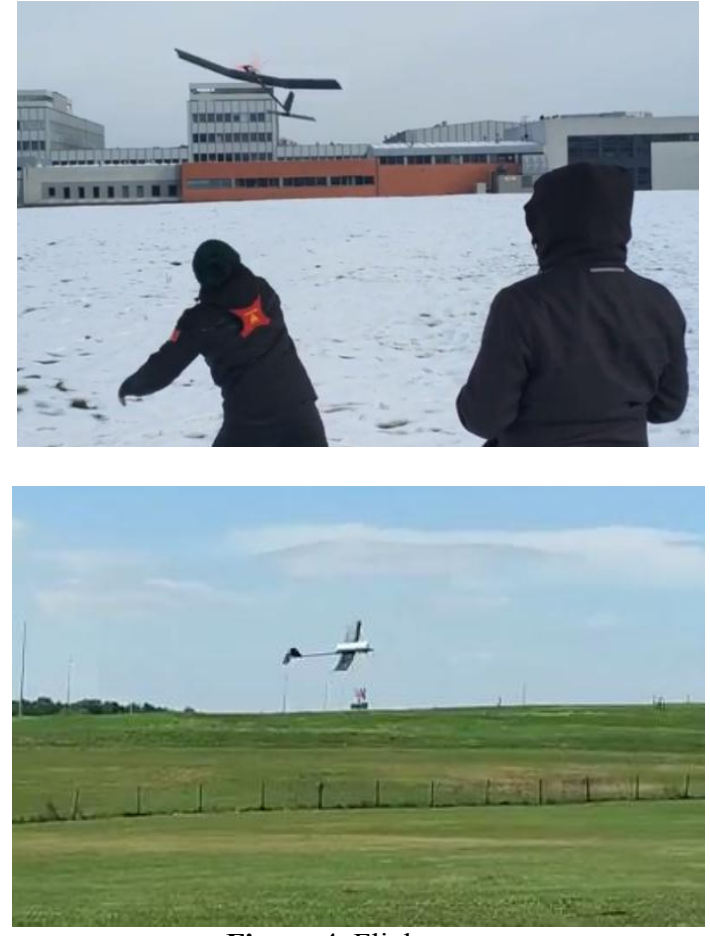

Figure 4. Flight tests

\section{Conclusions}

This paper covers development of geometry and innovative solutions of the light and compact unmanned aerial vehicle, which are optimal for given tasks and meet the initial requirements. Many different ideas were discussed, a compromise between theoretical and practical approaches was reached and the safety boundary was set to be sure that the aircraft can successful fly in any conditions. In addition to that, new tools for aircraft geometry optimization and mass model calculations were developed. To design the best possible UAV many tests, like dynamic thrust of the propulsion system and tensile testing, were done. Innovative manufacturing system was also developed to make as light and durable airplane as possible.

\section{References}

1. R. Austin Unmanned Aircraft Systems 45-74 (John Wiley and Sons 2010)

2. G. Landolfo Aerodynamic and structual design of a small nonplanar wing UAV 6-10, 35-67 (University of Dayton 2008)

3. B. W. McCormick Aerodynamics aeronautics, and flight mechanics (John Wiley \& Sons, 1979)

4. L. M. Nicolai Estimating $R / C$ model aerodynamics and performance VI (Lockheed Martin Aeronautical Company 2009)

5. T. C. Witushynsky Experimental Investigation into the aerodynamics of small air vehicles with thin flexible wings, 16-28 (Case Western Reserve University 2008)

6. T. J. Mueller, J. D. DeLaurier Aerodynamics of Small Vehicles 35: 89-111 (Annual Review of Fluid Mechanics 2003) 
7. T. Melin User's guide and reference manual for Tornado (KTH 2000)

8. W. Fiszdon Mechanika lotu 2 (P.W.N. 1961)

9. A. Skarbinski, W. Stafiej Projektowanie i konstrukcja szybowcow (Wydawnictwo Komunikacji i Lacznosci 1966)
10.P. G. Ifju Flexible-wing-based micro air vehicles 8 (Mechanical \& Aerospace Engineering Department, University of Florida 2005) 